\title{
Author Correction: Detection of anaemia from retinal fundus images via deep learning
}

Akinori Mitani (1), Abigail Huang, Subhashini Venugopalan (D), Greg S. Corrado, Lily Peng, Dale R. Webster (D), Naama Hammel, Yun Liu (1) and Avinash V. Varadarajan

Correction to: Nature Biomedical Engineering https://doi.org/10.1038/s41551-019-0487-z, published online 23 December 2019.

In the version of this Article originally published, in the sentence beginning "In a validation dataset of 11,388 study participants..., the terms "fundus-image-only" and "metadata-only" were in the wrong order; instead, the sentence should have read "In a validation dataset of 11,388 study participants from the UK Biobank, the metadata-only, fundus-image-only and combined models predicted haemoglobin concentration (in $\mathrm{g} \mathrm{dl}^{-1}$ ) with mean absolute error values of 0.73 (95\% confidence interval: $\left.0.72-0.74\right), 0.67(0.66-0.68)$ and 0.63 (0.62$0.64)$, respectively, and with areas under the receiver operating characteristic curve (AUC) values of 0.74 (0.71-0.76), 0.87 (0.85-0.89) and 0.88 (0.86-0.89), respectively." This has now been corrected.

Published online: 12 February 2020

https://doi.org/10.1038/s41551-020-0530-0

(C) The Author(s), under exclusive licence to Springer Nature Limited 2020 